\title{
Increasing the coherence time of Bose-Einstein-condensate interferometers with optical control of dynamics
}

\author{
James A. Stickney, ${ }^{1}$ Dana Z. Anderson, ${ }^{2}$ and Alex A. Zozulya ${ }^{1}$ \\ ${ }^{1}$ Department of Physics, Worcester Polytechnic Institute, \\ 100 Institute Road, Worcester, Massachusetts 01609, USA \\ ${ }^{2}$ Department of Physics and JILA, University of Colorado \\ and National Institute of Standards and Technology, \\ Boulder, Colorado 80309-0440, USA
}

\begin{abstract}
Atom interferometers using Bose-Einstein condensate that is confined in a waveguide and manipulated by optical pulses have been limited by their short coherence times. We present a theoretical model that offers a physically simple explanation for the loss of contrast and propose the method for increasing the fringe contrast by recombining the atoms at a different time. A simple, quantitatively accurate, analytical expression for the optimized recombination time is presented and used to place limits on the physical parameters for which the contrast may be recovered.
\end{abstract}

PACS numbers: 03.75.Dg, 39.20.+q, 03.75.Kk 


\section{INTRODUCTION}

The realization of a practical chip-based atom interferometer, using a Bose-Einstein condensate, (BEC) would revolutionize internal navigation systems, precision measurements, and, perhaps, quantum information technology. The production and manipulation of a condensate using an atom chip is appealing for many reasons. The trapping potentials, for the same electrical current, have a much higher frequency then their larger scale counterparts [1]. As a result, these devices require less power. An atomic cloud confined in a trap with a large frequency can be cooled to ultra-cold temperatures more rapidly [2], making the realization of a quasi-continuous source of condensed atoms more feasible. Chips also enable integration of several relatively simple components into devices with complex functionality $[3,4]$. Finally, the entire device, including the vacuum chamber, can be miniaturized [5], making chip-based devices the most likely path to practical applications.

The first experimentally realized BEC-based interferometers used a double-well potential to manipulate the atoms $[6,7]$. In this type of interferometer, atoms are initially prepared in a single potential well that confines the atoms in all three directions. Then the well is continuously transformed into a symmetric double well, which must be done adiabatically slow to avoid collective excitations. After the splitting, the wells are physically separated and, due to differences in the local environment, a quantum phase shift may develop between the atoms in each well. Nonlinearities, caused by atom-atom interactions, usually cause problems at the recombination stage in these types of interferometers. As a result, the atoms are recombined by suddenly switching off the trap allowing the two condensates to ballistically expand, overlap, and interfere. The phase shift can be measured by fitting the atomic density to a cos function.

An alternative method for realizing a BEC-based atom interferometer uses a standing light wave created by two counter-propagating laser pulses to manipulate the condensate [8-10]. The BEC is loaded into a waveguide providing confinement along two directions but not in the third one. The standing light wave splits the BEC into two harmonics moving in opposite directions. A second laser pulse reflects the harmonics by reversing their momentum. Finally, a third pulse recombines them, thus completing the interferometer sequence. Since the atoms are recombined in the guide, the phase shift between the two arms of the interferometer can be measured by counting the atoms in the zero momentum 
state at the end of the interferometer cycle.

In the first experimental realization of this type of interferometer [8], the coherence time was about $10 \mathrm{~ms}$. The loss of coherence was theoretically explained as being due to distortion of the phase across each atomic cloud [11], which is caused by both the atom-atom interactions and the residual external parabolic potential in the axial direction.

A similar interferometer with a coherence time of $44 \mathrm{~ms}$ has been realized in Ref. [9]. The coherence time of this interferometer was increased in two ways. First, the confining waveguide was created using macroscopic conductors, instead of microscopic conductors in a chip-based device. This larger scale device had looser confinement thus reducing the effect of the atom-atom interactions. Additionally, the waveguide was farther from the conductors minimizing small perturbations due to defects in the conductors or instabilities in the current. Second, the interferometer sequence used two reflections, such that each half of the condensate went through the same path twice in opposite directions. This method greatly reduces the phase distortion caused by the residual axial potential.

A third interferometer was recently realized in [12]. This chip-based device has a coherence time of about $15 \mathrm{~ms}$ and uses a sightly different splitting technique. One of the lasers is frequency-shifted with respect to the other laser, resulting in a traveling wave optical potential. Using a $\pi / 2$ pulse, the condensate is split into two harmonics one of which remains at rest and the other propagates with the momentum $2 \hbar k_{l}$. A $\pi$ pulse acts as a mirror, and finally a second $\pi / 2$ recombines the condensate. As a result of this different splitting mechanism, the splitting and recombination does not take place in the same physical location and the device is an atom Mach-Zehnder interferometer. By varying the radial confinement of the waveguide, the researchers were able to change the effective strength of the atom-atom interactions. They used this to test the theoretical model of Ref. [11] and demonstrated its validity.

In this paper, we discuss possible ways to increase the coherence time of an atom interferometer using Bragg diffraction to manipulate the atoms. Simple analytical expressions for the dynamics of the interference sequence are derived in the framework of the hydrodynamic approximation. Their validity is confirmed by their comparison with direct numerical solution of the Gross-Pitaevskii equation. We demonstrate that the coherence time can be increased by changing the recombination time and present simple analytic expressions for the optimized recombination time and the contrast. 
In the rest of the section, we present a simple physical explanation for the loss of contrast in a BEC Michelson interferometer and discuss possible ways of restoring it.

The interferometric cycle of duration $T$ starts by illuminating the motionless BEC cloud $\psi_{0}$ with a splitting pulse from a pair of counter- propagating laser beams. This pulse acts like a diffraction grating splitting the cloud into two harmonics $\psi_{+}$and $\psi_{-}$. The atoms diffracted into the +1 order absorb a photon from a laser beam with the momentum $\hbar k_{l}$ and re-emit it into the beam with the momentum $-\hbar k_{l}$ acquiring the net momentum $2 \hbar k_{l}$. The cloud $\psi_{+}$starts moving with the velocity $v_{0}=2 \hbar k_{l} / M$, where $k_{l}$ is the wavenumber of the laser beams and $M$ is the atomic mass. Similarly, the cloud $\psi_{-}$starts moving with the velocity $-v_{0}$. The two harmonics are allowed to propagate for the time $T / 2$ and are illuminated by a reflection optical pulse. The atoms in the harmonics $\psi_{+}$change their velocity by $-2 v_{0}$ and those in the harmonics $\psi_{-}$by $2 v_{0}$. The harmonics propagate back for time $T / 2$ and are subject to the action of the recombination optical pulse. After the recombination, the atoms in general populate all three harmonics $\psi_{0}$ and $\psi_{ \pm}$. The degree of population depends on the relative phase between the harmonics $\psi_{ \pm}$acquired during the interferometric cycle and can be used to deduce this phase. In particular, the wave function of the zero-momentum harmonics $\psi_{0}$ after the recombination is equal to

$$
\psi_{0}=\frac{1}{\sqrt{2}}\left(\psi_{+}+\psi_{-}\right)
$$

where $\psi_{ \pm}$are the wave functions of the \pm 1 harmonics immediately before the recombination.

Because of the nonlinearity and/or the external potential, the harmonics $\psi_{ \pm}$do not travel with the velocities $\pm v_{0}$ during the cycle. First, the cloud "climbing up" the external potential slows down and the one moving "downhill" speeds up. Second, because of the nonlinearity, the speeds of the two clouds after their separation will be slightly larger than $v_{0}$ if the nonlinearity is repulsive and slightly less than $v_{0}$ if it is attractive. For definiteness, we shall discuss the influence of the repulsive nonlinearity assuming that the external potential is zero. An ideal operation of the interferometer in this case corresponds to all the atoms populating zero-momentum harmonics $\psi_{0}$ after the recombination, i.e., to $N_{0}=N_{t o t}$.

Because of the atom-atom interaction, the clouds $\psi_{ \pm}$exert a repulsive force on each other during the time they overlap. This force accelerates each cloud so that after the separation pulse the $\psi_{ \pm}$harmonics propagate with velocities $\pm\left(v_{0}+\delta v\right)$, where $\delta v>0$. The reflection pulses impart the momenta $\mp 4 \hbar k_{l}$ to the clouds transforming their roles: $\psi_{ \pm} \rightarrow \psi_{\mp}$. After 
the reflection, the \pm 1 harmonics propagate with the velocities $\pm\left(v_{0}-\delta v\right)$. Harmonics' deceleration due to mutual repulsion during their overlap decreases the velocity of each harmonics by an additional $\delta v$ so immediately before the recombination the harmonics' velocities are $\pm\left(v_{0}-2 \delta v\right)$.

The nonzero value of $\delta v$ results in two consequences. First, since the clouds' speeds after the reflection pulse are smaller than before the pulse, the \pm 1 harmonics at the nominal recombination time still do not overlap each other completely. This effect is typically not very significant. Much more important is the fact that the returning harmonics have momenta that are not equal to $\pm 2 \hbar k_{l}$ and therefore can not be compensated by the recombination pulse. As a result, the wave function of the zero-momentum harmonics $\psi_{0}$ after the recombination can be written as

$$
\psi_{0} \propto \sqrt{n(x)} \cos (\Delta k x)
$$

where $\Delta k=2 M \delta v / \hbar$ and $n(x)$ are the density profiles of the harmonics; their possible incomplete overlap has been neglected. The population of the zero-momentum harmonics is obtained by the spatial integration of $\left|\psi_{0}\right|^{2}$. For $\Delta k R \ll 1$, where $R$ is the characteristic size of the clouds, all the atoms after the recombination are indeed in the zeroth harmonics, i.e. $N_{0}=N_{\text {tot }}$. In the opposite case $\Delta k R \gg 1$, the cos function oscillates several times across the cloud and $N_{0} / N_{\text {tot }}=1 / 2$ resulting in the loss of contrast. It is worth noting that the accumulation of corrections to the wave vectors of the clouds is due to the fact that the reflecting pulses do not reverse the clouds' velocities but rather add a constant velocity $\pm 2 v_{0}$ to them. This explains the fact that the coherence may be lost due to the presence of an external potential even when the nonlinearity is negligible.

The above-discussed loss of coherence due to incomplete cancelation of the wave vectors of the harmonics by the recombination pulse can be also visualized in the following way: the wave functions of the $\psi_{ \pm}$harmonics can be represented as $\psi_{ \pm}=\sqrt{n_{ \pm}(x)} \exp \left(i \phi_{ \pm}\right)$, where $\phi_{ \pm}$ is the parabolic phase (the nominal phase $\pm\left(M v_{0} / \hbar\right) x$ is taken care of by the optical pulses and is not included). In the ideal situation, the parabolic phase for each cloud is centered at the middle of the cloud. Nonzero values of $\delta v$ (or, equivalently, nonzero values of the corrections to the wave vectors of the clouds) mean that the phase of each cloud leads or lags behind its density envelope. This situation is schematically illustrated in Fig. 1 showing the harmonic $\psi_{+}$before the recombination with its phase leading the density envelope. We 
shall show that the optimum recombination corresponds to the situation when the phase profiles, not the density envelopes of the clouds are on top of each other immediately before the recombination.

Operation with high values of the contrast can be achieved in several ways. First, the relative magnitudes of the nonlinearity and the external potential are adjusted in such a way that their effects cancel each other for a given cycle time $T$ (this is not always possible). Second, the recombination and/or reflection are conducted with optical pulses having different wavelength as compared to the splitting pulse to compensate for the change in the wave vectors of the moving clouds. Finally, the recombination is carried out not at the nominal recombination time $T$ but at a time such that $\Delta k R=0$. The paper is devoted to the analysis of the last possibility.

The rest of the paper is structured as follows: Sec. II provides general formulation of the problem, Sec. III introduces analytical model of the interferometric cycle and Sec. IV is devoted to the analysis of the contrast and contains the analytical expressions for the optimized recombination time. These expressions are discussed in different limiting cases in Sec. V.

\section{FORMULATION OF THE PROBLEM}

The evolution of the condensate in the interferometer in the mean-field limit is described by the Gross-Pitaevskii equation

$$
i \hbar \frac{\partial}{\partial t} \Psi(\boldsymbol{r}, t)=\left(-\frac{\hbar^{2}}{2 M} \nabla^{2}+V_{t o t}(\boldsymbol{r}, t)+U_{0} N|\Psi|^{2}\right) \Psi(\boldsymbol{r}, t),
$$

where $\Psi(\boldsymbol{r}, t)$ is the wave function of the condensate that is normalized to one, $N$ is the total number of atoms, $U_{0}=4 \pi \hbar^{2} a_{s} / M$ characterizes the strength of interatomic interactions, $a_{s}$ is the s-wave scattering length and $M$ is the atomic mass. The potential $V_{t o t}=V_{3 D}(\boldsymbol{r}, t)+$ $V_{\text {opt }}(t) \cos \left(2 k_{l} x\right)$ is the sum of a confining potential $V_{3 D}$ and an optical potential that is created by two counter-propagating laser beams of wavelength $\lambda=2 \pi / k_{l}$, which are detuned from the atomic resonance to avoid spontaneous emission. The optical potential is used to split, recombine and reverse direction of propagation of the BEC clouds.

The confining potential is of the form

$$
V_{3 D}(\boldsymbol{r}, t)=V(x, t)+M \omega_{\perp}^{2} r_{\perp}^{2} / 2,
$$


where $V(x, t)$ is slowly-spatially-varying potential due to the environment and $M \omega_{\perp}^{2} r_{\perp}^{2} / 2$ is the guiding potential providing confinement of the condensate along the two spatial dimensions $\boldsymbol{r}_{\perp}=(y, z)$. In the following we shall assume that the condensate is tightly confined in the two transverse dimensions and is in the lowest transverse mode of the guide

$$
\psi_{\perp}\left(r_{\perp}\right)=\frac{1}{\sqrt{\pi} a_{\perp}} \exp \left(-r_{\perp}^{2} / 2 a_{\perp}^{2}\right)
$$

where $a_{\perp}=\left(\hbar / M \omega_{\perp}\right)^{1 / 2}$ is the transverse oscillator length. Factorizing the wave function of the condensate as $\Psi(\boldsymbol{r}, t)=\psi(x, t) \psi_{\perp}\left(r_{\perp}\right)$, Eq. (3) can be reduced to the one-dimensional equation for the function $\psi(x, t)$. Introducing dimensionless coordinate $x \rightarrow 2 k_{l} x$ and time $\tau=t / t_{0}$, where $t_{0}=M /\left(4 \hbar k_{l}^{2}\right)$, this one-dimensional Gross-Pitaevskii equation can be written as

$$
i \frac{\partial}{\partial \tau} \psi(x, \tau)=\left[-\frac{1}{2} \frac{\partial^{2}}{\partial x^{2}}+v(x, \tau)+\Omega(\tau) \cos (x)+p|\psi|^{2}\right] \psi(x, \tau),
$$

where $v=(V / \hbar) t_{0}, \Omega=\left(V_{\text {opt }} / \hbar\right) t_{0}$ and $p=a_{s} N / a_{\perp}^{2} k_{l}$.

The optical potential $\Omega(\tau) \cos x$ acts as a diffraction grating for the condensate wave function $\psi$. This grating diffracts the condensate into several harmonics separated by multiples of the grating wavevector. If the width of the Fourier spectrum of the condensate is much smaller than the length of the grating wavevector (one in our dimensional units), the wave function $\psi(x, t)$ in Fourier space consists of a series of narrow peaks. It is therefore convenient to represent $\psi(x, t)$ as

$$
\psi(x, t)=\sum_{n} \psi_{n}(x, t) \exp (i n x)
$$

where harmonics' envelopes $\psi_{n}(x)$ are slowly-varying functions of coordinate as compared with the exponentials. The dynamics of these harmonics are governed by the set of coupled equations

$$
\begin{aligned}
& i\left(\frac{\partial}{\partial \tau}+i n \frac{\partial}{\partial x}\right) \psi_{n}=\frac{1}{2}\left(-\frac{\partial^{2}}{\partial x^{2}}+n^{2}\right) \psi_{n}+v(x, \tau) \psi_{n} \\
& +\frac{\Omega(\tau)}{2}\left(\psi_{n+1}+\psi_{n-1}\right)+p \sum_{l, m} \psi_{l}^{*} \psi_{m} \psi_{n-m+l}
\end{aligned}
$$

The optical potential $\Omega(\tau)$ in Eq. (6) is used to split the initial zero-momentum BEC cloud at the beginning of the interferometric cycle into the two harmonics with the momenta \pm 1 , reverse their direction of propagation in the middle of the cycle and recombine 
them at the end. Dynamics of the BEC due to the optical potential was fully taken into account in solving the Gross-Pitaevskii equation numerically. In the analytical model, their

action was described in terms of simple transformation matrices. For self-consistency of the presentation, a brief derivation of parameters of optical pulses used in the numerical solution of the Gross-Pitaevskii equation is given in the Appendix. The results presented in the Appendix have been previously derived in Refs. [9, 10, 13]. The next section is devoted to the development of analytical model describing evolution of the BEC between the optical pulses.

\section{PARABOLIC MODEL}

Between the optical pulses the condensate consists of two harmonics with $n= \pm 1$ whose evolution is described by the set of coupled equations

$$
i\left(\frac{\partial}{\partial \tau} \pm \frac{\partial}{\partial x}\right) \psi_{ \pm}=-\frac{1}{2} \frac{\partial^{2}}{\partial x^{2}} \psi_{ \pm}+v(x) \psi_{ \pm}+p\left(\left|\psi_{ \pm}\right|^{2}+2\left|\psi_{\mp}\right|^{2}\right) \psi_{ \pm} .
$$

Introducing the density and phase of each harmonic by the relations

$$
\psi_{ \pm}=\sqrt{n_{ \pm}} \exp \left(i \phi_{ \pm}\right)
$$

and using the Thomas-Fermi approximation (neglecting the second derivatives of the density) transforms the set of equations (9) to the form

$$
\begin{aligned}
& \left(\frac{\partial}{\partial \tau} \pm \frac{\partial}{\partial x}\right) n_{ \pm}=-\frac{\partial}{\partial x}\left(n_{ \pm} \frac{\partial \phi_{ \pm}}{\partial x}\right) \\
& \left(\frac{\partial}{\partial \tau} \pm \frac{\partial}{\partial x}\right) \phi_{ \pm}=-\frac{1}{2}\left(\frac{\partial \phi_{ \pm}}{\partial x}\right)^{2}-v-p\left(n_{ \pm}+2 n_{\mp}\right) .
\end{aligned}
$$

We will describe the external potential $v$ by the first two terms of the Taylor expansion

$$
v(x)=\alpha x+\frac{1}{2} \beta x^{2}
$$

and analyze the set of Eq. (10) in the framework of a parabolic approximation where expressions for both the density and the pase do not contain terms higher than the second order in coordinate:

$$
\begin{aligned}
& n_{ \pm}=\frac{3}{8 R}\left[1-\frac{\left(x-x_{ \pm}\right)^{2}}{R^{2}}\right] \\
& \phi_{ \pm}=\varphi_{ \pm}+\kappa_{ \pm}\left(x-x_{ \pm}\right)+\frac{g}{2}\left(x-x_{ \pm}\right)^{2} .
\end{aligned}
$$


$R, x_{ \pm}, \kappa_{ \pm}, \varphi_{ \pm}$and $g$ are functions of time $\tau$ only. The coefficient $3 / 8$ in the expression for $n_{ \pm}$follows from the normalization condition (each harmonics is normalized to $1 / 2$ ). Note that for each cloud its density and phase in Eq. (12) are defined only in the region where the density is nonnegative. Functions $x_{ \pm}(\tau)$ are positions of the centers of mass of the two moving clouds, $\kappa_{ \pm}$are corrections to their nominal wavevectors $( \pm 1)$ that are due to the external potential and the nonlinearity and $\varphi_{ \pm}$are the accumulated coordinate-independent phases. Finally, $R$ is the half-size of each of the clouds and the parameter $g$ multiplying the quadratic part of the phase is analogous to the inverse of the radius of curvature of the wavefront of a propagating light beam in optics.

Using Eq. (12) and the first of Eq. (10), one gets

$$
\begin{aligned}
& R^{\prime}=g_{ \pm} R, \\
& x_{ \pm}^{\prime}= \pm 1+\kappa_{ \pm},
\end{aligned}
$$

where the prime means differentiation with respect to time. Treatment of the second Eq. (12) is slightly complicated by the fact that the regions of existence of $n_{+}$and $n_{-}$do not coincide. Since the functional forms of $n$ and $\phi$ are fixed, the density profile $n_{\mp}$ should be projected onto $n_{ \pm}$. To do this, one can choose a set of suitable basis functions defined at the interval $\left|\xi_{ \pm}\right| \leq 1$, where $\xi_{ \pm}=\left(x-x_{ \pm}\right) / R$, that can be used to represent the density $n_{ \pm}$and the phase $\phi_{ \pm}$. The density $n_{\mp}$ should then be expressed in terms of the same basis set retaining only the functions that describe $n_{ \pm}$and $\phi_{ \pm}$. Using Legendre polynomials $P_{n}\left(\xi_{ \pm}\right)$as the basis yields

$$
\frac{16 R}{3} n_{\mp}\left(\xi_{ \pm}\right) \rightarrow d_{0} \mp d_{1} \xi_{ \pm}-d_{2} \xi_{ \pm}^{2},
$$

where

$$
\begin{aligned}
& d_{0}=\left(2-\frac{7}{2}|q|^{2}+2|q|^{3}-\frac{1}{8}|q|^{5}\right) \theta(|q|<2), \\
& d_{1}=q\left(4-3|q|+\frac{1}{4}|q|^{3}\right) \theta(|q|<2), \\
& d_{2}=\left(2-\frac{15}{2}|q|^{2}+5|q|^{3}-\frac{3}{8}|q|^{5}\right) \theta(|q|<2)
\end{aligned}
$$

and $q=\left(x_{+}-x_{-}\right) / R$. The $\theta$-function in Eq. (15) is equal to one if its argument is a logical true and zero if its is a logical false.

Using Eq. (14) in the second of Eq. (10) yields equations of motion for $g, \kappa_{ \pm}$and $\varphi_{ \pm}$. 
Combining these with Eq. (13), we get the final set of equations

$$
\begin{aligned}
& R^{\prime}=g R \\
& g^{\prime}=-g^{2}-\beta+\frac{3 p}{4 R^{3}}\left(1+d_{2}\right), \\
& \kappa_{ \pm}^{\prime}=-\alpha-\beta x_{ \pm} \pm \frac{3 p}{8 R^{2}} d_{1}, \\
& x_{ \pm}^{\prime}= \pm 1+\kappa_{ \pm}, \\
& \varphi_{ \pm}^{\prime}=\frac{\kappa_{ \pm}^{2}}{2}-\alpha x_{ \pm}-\frac{1}{2} \beta x_{ \pm}^{2}-\frac{3 p}{8 R}\left(1+d_{0}\right),
\end{aligned}
$$

where prime means differentiation with respect to time. Equations (16) have simple physical interpretation. The rates of change of the coordinates of the two clouds $\psi_{ \pm}$are given by the relations $x_{ \pm}^{\prime}= \pm 1+\kappa_{ \pm}$, i.e., the clouds move with velocities $\pm 1+\kappa_{ \pm}$. The major contributions to the velocities \pm 1 are due to the momenta imparted to the clouds by the optical pulses. The corrections $\kappa_{ \pm}$are due to the external potential (parameters $\alpha$ and $\beta$ ) and the nonlinearity. The cloud "climbing up" the external potential slows down and the one moving "downhill" speeds up. If the nonlinearity is repulsive $(p>0)$, the speeds of the two clouds after their separation will be slightly larger than one and if it is attractive, slightly less than one. The functions $d_{0}, d_{1}$ and $d_{2}$ given by Eq. (15) describe mutual interaction of the two clouds. They depend on the relative displacement of the clouds $q=\left(x_{+}-x_{-}\right) / R$

and are nonzero only when $|q|<2$, i.e., when the clouds overlap. The other terms containing the nonlinearity parameter $p$ describe self interaction for each of the clouds and are always nonzero.

\section{A. Evolution of $\kappa_{ \pm}$and $x_{ \pm}$}

During the interferometric cycle the two BEC clouds $\psi_{ \pm}$may be partially overlapping or non-overlapping. In the subsequent analysis, it will be assumed that the size of each cloud does not change significantly at the time intervals $\tau \propto R$ that it takes for the clouds to pass each other. The conditions of applicability of this assumption are given by Eq. (29). Additionally, it will be assumed that $\beta T^{2} \ll 1$, where $T$ is the duration of the interferometric cycle. 
Time evolution of $\kappa_{ \pm}$and $x_{ \pm}$is governed by the set of two coupled equations (cf. (16))

$$
\begin{aligned}
& \kappa_{ \pm}^{\prime}=-\alpha-\beta x_{ \pm} \pm \frac{3 p}{8 R^{2}} d_{1}, \\
& x_{ \pm}^{\prime}= \pm 1+\kappa_{ \pm},
\end{aligned}
$$

Solution of Eqs. (17) can be written as

$$
\begin{gathered}
\kappa_{ \pm}(\tau)=\kappa_{ \pm, 0}-\alpha \tau-x_{ \pm, 0} \beta \tau \mp \frac{1}{2} \beta \tau^{2} \pm \frac{3 p}{16 R} \int_{q_{0}}^{q_{0}+2 \tau / R_{0}} d q d_{1}(q), \\
x_{ \pm}(\tau)=x_{ \pm, 0}+\left( \pm 1+\kappa_{ \pm, 0}\right) \tau-\frac{1}{2} \alpha \tau^{2}-\frac{1}{2} x_{ \pm, 0} \beta \tau^{2} \mp \frac{1}{6} \beta \tau^{3} \\
\pm \frac{3 p}{32} \int_{q_{0}}^{q_{0}+2 \tau / R_{0}} d q \int_{q_{0}}^{q} d q^{\prime} d_{1}\left(q^{\prime}\right),
\end{gathered}
$$

where $\kappa_{ \pm, 0}, x_{ \pm, 0}$ and $R_{0}$ are initial values of $\kappa_{ \pm}, x_{ \pm}$and $R$. In deriving Eq. (18) and (19), the dynamics of the relative separation between the clouds in evaluating function $d_{1}$ was approximated by the relation

$$
q(\tau)=q_{0}+\frac{2 \tau}{R_{0}}
$$

i.e., the terms with $\kappa_{ \pm}$were neglected as compared to one in evaluating $q(\tau)$.

The interferometric cycle of duration $T$ starts by applying the splitting optical pulses to the motionless cloud $\psi_{0}$, letting harmonics $\psi_{ \pm}$propagate for the time $T / 2$, reverse their directions of propagation by applying the reflection pulses, letting the harmonics $\psi_{ \pm}$evolve for the time $T / 2$ and apply the recombination optical pulses.

Immediately after the splitting pulses at $\tau=0$, the center of mass of each harmonic is $x_{ \pm, 0}=0, q_{0}=0$ and $\kappa_{ \pm, 0}=0$. The reflection pulse reverses directions of propagation of the two harmonics by adding momenta $\mp 2$ to the momenta $\pm 1+\kappa_{ \pm}$of $\psi_{ \pm}$. After the reflection pulse the harmonic $\psi_{+}$becomes $\psi_{-}$and vice versa. As a result, immediately after the reflection pulse, $x_{ \pm, 0}=x_{\mp}(T / 2)$ and $\kappa_{ \pm, 0}=\kappa_{\mp}(T / 2)$.

At the nominal recombination time $\tau=T$, the corrections to the velocities $\kappa_{ \pm}$and the center of mass coordinates $x_{ \pm}$are given by the relations

$$
\begin{gathered}
\kappa_{ \pm}(T)=-\alpha T \pm \frac{1}{4} \beta T^{2} \mp p\left[\frac{1}{R_{0}} D_{1}\left(T / R_{0}\right)+\frac{1}{R_{T}} D_{1}\left(T / R_{T}\right)\right], \\
x_{ \pm}(T)=-\frac{1}{2} \alpha T^{2} \pm \frac{1}{8} \beta T^{3} \\
\mp \frac{p}{2}\left[\int_{T / R_{T}}^{T / R_{0}} d q D_{1}(q)+\frac{T}{R_{0}} D_{1}\left(T / R_{0}\right)+\frac{T}{R_{T}} D_{1}\left(T / R_{T}\right)\right],
\end{gathered}
$$


where

$$
D_{1}(x)=\left\{\begin{array}{cc}
\frac{3}{16} x^{2}\left(2-x+\frac{1}{20} x^{3}\right) & , x<2 \\
D_{1}(2)=3 / 10 & , x>2,
\end{array}\right.
$$

$R_{0}$ is the size of the harmonics at the separation stage and $R_{T}$ is the size during recombination.

\section{B. Evolution of $g$ and $R$}

Evolution of $g$ and $R$ is governed by the set of two coupled equations (see Eq. (16))

$$
\begin{aligned}
& R^{\prime}=g R, \\
& g^{\prime}=-g^{2}-\beta+\frac{3 p}{4 R^{3}}\left(1+d_{2}\right) .
\end{aligned}
$$

The explicit expressions for $g$ and $R$ at time intervals $\tau$ such that $R$ does not change significantly, i.e., $|\Delta R| \ll R$, are of the form

$$
\begin{gathered}
g(\tau)=g_{0}-\beta \tau+\frac{3 p}{8 R_{0}^{2}} \int_{q_{0}}^{q_{0}+2 \tau / R_{0}} d q\left[1+d_{2}(q)\right], \\
R(\tau)=R_{0}+R_{0}\left[g_{0} \tau-\frac{\beta}{2} \tau^{2}+\frac{3 p}{8 R_{0}^{2}} \int_{0}^{\tau} d \tau^{\prime} \int_{q_{0}}^{q_{0}+2 \tau^{\prime} / R_{0}} d q\left[1+d_{2}(q)\right]\right],
\end{gathered}
$$

where $g_{0}=g(0), q_{0}=q(0)$ and $R_{0}=R(0)$ are initial values of $g, q$ and $R$. In deriving Eqs. (25) and (26), the terms of the order $\kappa_{ \pm}$in the equations for $x_{ \pm}$have been neglected as compared to one. The dynamics of the relative separation between the clouds in the framework of this approximation is given by the expression

$$
q(\tau)=q_{0}+\frac{2 \tau}{R_{0}}
$$

Equations (25) and (26) are valid provided

$$
g_{0} \tau, \beta \tau^{2}, \frac{p \tau^{2}}{R_{0}^{3}} \ll 1
$$

In the analysis of Sec.III A and in the rest of the paper it is assumed that the size of each cloud does not change significantly during the time $\tau=R$ that it takes for the clouds to pass each other. The conditions of applicability of this approximation are

$$
g_{0} R_{0}, \beta R_{0}^{2}, \frac{p}{R_{0}} \ll 1
$$


Using Eq. (25), we get the following expression for the value of $g$ at the recombination time in the limit when $R$ does not change significantly during the interferometric cycle:

$$
g(T)=g_{0}-\beta T+\frac{3 p T}{4 R^{3}}+\frac{p}{R^{2}} D_{2}(T / R)
$$

where

$$
D_{2}(x)=\left\{\begin{array}{cc}
\frac{3}{4} x\left(2-\frac{5}{2} x^{2}+\frac{5}{4} x^{3}-\frac{1}{16} x^{5}\right) & , x<2, \\
D_{2}(2)=0 & , x>2
\end{array}\right.
$$

The limit $|\Delta R| \ll R$ can correspond to both $\tau<R$ when the clouds stay overlapped during all the cycle and to $\tau \gg R$ when they do not overlap most of the cycle. The second limit of interest to be considered in this section $\tau \gg R$ explicitly deals with the situation when the clouds do not overlap most of the time. In this limit, the contribution coming from the function $d_{2}$ in Eq. (24) (interaction between the clouds) can be neglected as compared to their self action. The function $g$ in this limit is given by the relation

$$
g(\tau)=\frac{1}{r} \frac{d}{d \tau} r=\frac{\operatorname{sign} g_{0}}{r}\left[g_{0}^{2}-\frac{3 p}{2 R_{0}^{3}}\left(\frac{1}{r}-1\right)-\beta\left(r^{2}-1\right)\right]^{1 / 2},
$$

where $r=R(\tau) / R_{0}$. Note that Eq. (32) is valid for any values of $R(\tau)$. The general expression for $R(\tau)$ can be obtained in terms of elliptic integrals but is too cumbersome to be of practical use. In the limit where the relative change in the size of each harmonic is small $(|r-1| \ll 1)$, one gets

$$
\begin{aligned}
& R(\tau)=R_{0}+R_{0}\left[g_{0} \tau+\left(\frac{3 p}{4 R_{0}^{3}}-\beta\right) \frac{\tau^{2}}{2}\right], \\
& g(\tau)=g_{0}+\left(\frac{3 p}{4 R_{0}^{3}}-\beta\right) \tau .
\end{aligned}
$$

These expressions coincide with Eqs. (25) and (26) when $\tau \gg R$ and describe the situation when the clouds do not overlap most of the time but their sizes do not changes significantly during all their evolution time.

In the opposite limit $R(\tau) \gg R_{0}$,

$$
g(\tau)=\operatorname{sign} g_{0} \frac{R_{0}}{R(\tau)}\left[g_{0}^{2}+\frac{3 p}{2 R_{0}^{3}}-\beta \frac{R^{2}(\tau)}{R_{0}^{2}}\right]^{1 / 2}
$$




\section{Evolution of $\varphi_{ \pm}$}

In an interferometric experiment, the quantity of interest is not the absolute phase of each harmonic $\varphi_{ \pm}$, but rather the relative phase $\Delta \varphi=\varphi_{+}-\varphi_{-}$. The time evolution of $\Delta \varphi$ is governed by the equation

$$
\Delta \varphi^{\prime}=\frac{1}{2}\left(\kappa_{+}^{2}-\kappa_{-}^{2}\right)-\alpha\left(x_{+}-x_{-}\right)-\frac{1}{2} \beta\left(x_{+}^{2}-x_{-}^{2}\right)
$$

Using results of Section III A and neglecting terms containing products and quadratic or higher combinations of $\alpha, \beta$ and $p$ yields

$$
\Delta \varphi(T)=-\frac{\alpha}{2} T^{2}
$$

\section{THE INTERFERENCE SIGNAL}

The wavefunction of the zero-momentum harmonics after the recombination is given by the expression:

$$
\begin{aligned}
& \psi_{0}(x)=\frac{1}{\sqrt{2}}\left[\psi_{+}(x)+\psi_{-}(x)\right] \propto \frac{1}{\sqrt{2}}\left[\sqrt{n\left(x-x_{+}\right)} \exp \left(i \theta_{0} / 2+i \Delta k x / 2\right)\right. \\
& \left.+\sqrt{n\left(x+x_{+}\right)} \exp \left(-i \theta_{0} / 2-i \Delta k x / 2\right)\right] .
\end{aligned}
$$

Here $\psi_{ \pm}$are the wavefunctions of the \pm 1 harmonics before the recombination,

$$
\begin{gathered}
n(x)=\frac{3}{8 R}\left(1-\frac{x^{2}}{R^{2}}\right), \\
\theta_{0}=\left(\varphi_{+}-\varphi_{-}\right)-\left(\kappa_{+} x_{+}-\kappa_{-} x_{-}\right)+\frac{g}{2}\left(x_{+}^{2}-x_{-}^{2}\right)
\end{gathered}
$$

and

$$
\Delta k=\Delta \kappa-g \Delta x,
$$

where $\Delta \kappa=\kappa_{+}-\kappa_{-}$and $\Delta x=x_{+}-x_{-}$. All quantities in Eq. (39) are evaluated at the recombination time.

If the density envelopes of the \pm 1 harmonics sufficiently overlap at the recombination stage, Eq. (37) can be simplified to

$$
\psi_{0}(x)=\sqrt{n(x)} \cos \left(\theta_{0} / 2+\Delta k x / 2\right)
$$


Population of the zero-momentum harmonics $N_{0}$ is given by the spatial integration of $\left|\psi_{0}\right|^{2}$ yielding

$$
\frac{N_{0}}{N_{t o t}}=\frac{1}{2}\left(1+V \cos \theta_{0}\right),
$$

where the contrast of the interference fringes $V$ is given by the expression

$$
V=\frac{3}{(\Delta k R)^{3}}[\sin (\Delta k R)-\Delta k R \cos (\Delta k R)] .
$$

For $\Delta k R \ll 1$, the population of the zero-momentum state is given by the relation

$$
N_{0} / N_{\text {tot }}=\cos ^{2}\left(\theta_{0} / 2\right)
$$

In this limiting case the population depends on the relative accumulated coordinateindependent phase $\theta_{0}$ between the two $B E C$ clouds and exhibits interference fringes as a function of this phase.

In the opposite case $\Delta k R \gg 1$, the cos function in Eq. (41) oscillates several times across the cloud and

$$
N_{0} / N_{t o t}=1 / 2
$$

independently of the value of the relative phase shift.

Equations (22) and (21) show that both the nonlinearity of the condensate $p$ and the quadratic contribution to the external potential $\beta$ can result in nonzero values of $\Delta k$ given by Eq. (40) and thus be responsible for the loss of interferometric contrast as illustrated by Fig. 2. This figure shows the contrast $V$ defined by the relation $N_{0} / N_{t o t}=(1+V) / 2$, where $N_{0} / N_{\text {tot }}$ is the relative population of the zero-momentum harmonics at the end of the interferometric cycle, as a function of the ratio of the cycle time to the initial size of the harmonic $T / R_{0}$. The solid line corresponds to the numerical solution of the Gross-Pitaevskii equation (6). The dashed line is given by Eq. (43), where $\Delta k R$ is calculated with the help of analytical expressions (40), (21), (22) and (30).

Since the linear slope of the potential is zero $(\alpha=0), \theta_{0}=0$. Equation (43) then predicts that for $\Delta k R=0, V=1$. As is seen in Fig. 2, the contrast indeed equals one for short cycles (small $T$ ). Larger values of $T$ correspond to larger interaction times between the two clouds and an increase in $\Delta k R$ due to this interaction. As the interaction time increases, the contract $V$ given by Eq. (43) goes down from one to small negative value resulting in the values of $N_{0} / N_{\text {tot }}$ slightly below $1 / 2$. At times larger than about $T / R_{0}=1.5$ 
the two harmonics completely pass each other and stop overlapping during a part of the cycle. The interaction time between the harmonics (the time when they overlap) is now smaller than the cycle time and does not depend on it. The contrast and the population of the zero-momentum harmonic reach their limiting values. Figure 3 shows the dependence of the population of the zeroth-order harmonic after the recombination $N_{0} / N_{\text {tot }}$ on the relative accumulated phase shift $\theta_{0}=-\alpha T^{2} / 2$. The solid line corresponds to the numerical solution of the Gross-Pitaevskii equation (6). The dashed line is Eq. (42) with $\Delta k R$ given by Eqs. (21), (22) and (30). As is seen in Fig. 2, the cycle time $T / R_{0}=4$ corresponds to small negative values of the contrast $(V \approx-0.2$ as given by the Gross-Pitaevskii equation and $V \approx-0.1$ as given by the analytic model). Low values of the contrast result in the washout of the interference fringes shown in Fig. 3. It also should be noted that since the contrast $V$ is negative, the symmetric recombination with $\theta_{0}=0$ corresponds not to the maximum, but the minimum population $N_{0}$ of the zero-momentum harmonic.

Figures 2 and 3 demonstrate that recombination with nonzero value of the linear wavevector $\Delta k$ (see Eq. (40)) washes out the interference fringes. Since $\Delta k$ is a function of time, this effect can be compensated for by conducting the recombination not at the nominal time $T$ but at a slightly different time $T+\Delta T$ when $\Delta k R=0$ (in general, $\Delta T$ may be both positive and negative). Figure 4 shows the contrast $V=2 N_{0} / N_{t o t}-1$, where $N_{0} / N_{\text {tot }}$ is relative population of the zero-momentum harmonic, as a function of the time $\Delta T$. Negative (positive) values of $\Delta T$ correspond to the recombination taking place slightly before (after) the nominal recombination time $T$. The parameters for Fig. 4 are $T=2000, R_{0}=500$ and $p=5$ with all other parameters being zero. The solid line is the solution of the GrossPitaevskii equation and the dashed line is obtained with the help of Eqs. (42), (21), (22) and (32). Recombination at the nominal time $\Delta T=0$ corresponds to a small value of the contrast and a washout of the fringes as is shown in Fig. 3. Figure 4 indicates that if the recombination takes place at $\Delta T / R_{0} \approx-0.2$, the contrast of the fringes becomes much larger. This is confirmed by Fig. 5 , which shows $N_{0} / N_{\text {tot }}$ versus the relative accumulated phase shift $\theta_{0}=-\alpha\left[(T+\Delta T)^{2}-T^{2} / 2\right]$ for $\Delta / R=-0.2$ and all other parameters the same as in Fig. 3. The solid line is the solution of the Gross-Pitaevskii equation and the dashed line is the result of the numerical solution of Eqs. (16). 
The simple estimate using the condition $(\Delta k R)(T+\Delta T)=0$ yields

$$
\frac{\Delta T}{R_{T}}=-\frac{(\Delta k R)_{T}}{R(\Delta k R)_{T}^{\prime}}
$$

The ratio $\Delta T / R$ gives the relative displacement of the two clouds at the recombination time $T+\Delta T$ since the clouds pass across each other in time $R$ (each cloud has the size $2 R$ and the relative speed is 2).

The population of the zero-momentum harmonics depends not only on the magnitude of $\Delta k R$, but on the degree of overlap of the two density envelopes at the recombination time (see (37)). Estimate (44) takes into account only changes in $\Delta k R$ but not in the overlap in evaluating $\Delta T$. The last can be taken into account in the framework of Eq. (37) at the expense of making formulas more cumbersome and turn out to be not very significant. As we shall see, Eq. (44) is in a very good qualitative and quantitative agrement with the results of numerical solution of the Gross-Pitaevskii equation (6). Finally it should be mentioned that nonzero values of $\Delta T / R$ mean incomplete overlap and thus the contrast less than one even at the optimized time. The larger is $|\Delta T| / R$, the smaller the contrast. The estimate (44) implicitly implies that $|\Delta T| / R \leq 1$ because correction to the recombination time is meaningful only if the clouds overlap at the time $T+\Delta T$. If, for some set of parameters, estimate (44) yields $|\Delta T| / R>1$, the coherence can not be recovered for this set of parameters.

Using the explicit expressions for $(\Delta k R)$ and its time derivative obtained with the help of Eqs. (40) and (16) results in the relation

$$
\frac{\Delta T}{R}=\frac{\Delta \kappa-g \Delta x}{2 g R-3 p \Delta x / 4 R^{2}} .
$$

In Equation (45), $\Delta \kappa(T)$ and $\Delta x(T)$ are evaluated using Eq. (21) and (22). The function $g(T)$ should be evaluated using several different expressions depending on the parameters of the problem. For $|\Delta R| \ll R, g(T)$ is given by Eq. (30). In this case $R_{0}=R_{T}=R$. If $T \gg R_{0}, g(T)$ is given by Eq. (32). Since we are assuming that $|\Delta R| \gg R$ at times it takes the clouds to pass through each other, Eqs. (30) and (32) cover all possible situations. If $|\Delta R|$ is not small as compared to $R$, the size of the clouds $R_{T}$ at the end of the cycle should be evaluated by numerical integration of Eq. (32).

Equation (45) is relatively complex because it covers both the case when the size of the clouds does not change significantly during the cycle and the opposite limit when the final 
size is much larger than the initial one. All the relevant physics can be understood by discussing the case $|\Delta R| \ll R$ when Eq. (45) acquires especially simple form

$$
\frac{\Delta T}{R}=\frac{\Delta \kappa}{2 g R}=-\frac{1}{4} \frac{8 D_{1}(\zeta)-\left(\beta R^{3} / p\right) \zeta^{2}}{3 \zeta / 4+D_{2}(\zeta)+g_{0} R^{2} / p-\left(\beta R^{3} / p\right) \zeta},
$$

where $\zeta=T / R$.

The contrast at the optimized recombination time can be evaluated by accounting for an incomplete overlap of the clouds using Eq. 37 and is given by the approximate expression

$$
V \approx 1-\frac{3}{2}\left(\frac{\Delta T}{R}\right)^{2}\left[\ln \frac{R}{|\Delta T|}+2 \ln 2-\frac{1}{2}\right] .
$$

Equations (46) and (47) are the main analytical results of the paper. In Section V, they will be analyzed in several illustrative cases.

\section{DISCUSSION}

\section{A. Influence of the nonlinearity $p$ for $g_{0}=\beta=0$}

For $\beta=g_{0}=0$, Eq. (46) becomes

$$
\frac{\Delta T}{R}=\frac{-2 D_{1}(T / R)}{D_{2}(T / R)+3 T / 4 R}
$$

Equation (48) shows that the correction to the recombination time depends only on the

single parameter $T / R$ and does not depend on the nonlinearity of the condensate $p$ (the applicability of the parabolic approximation requires $p R \gg 1$ ). This is due to the fact that both the $\Delta \kappa$ and the $g R$ terms are proportional to the nonlinearity parameter $p$. At small values of $T / R, D_{1}(T / R) \approx(3 / 8)(T / R)^{2}, D_{2}(T / R) \approx(3 / 2)(T / R)$ (cf. Eqs. (23) and (31)) so

$$
\frac{\Delta T}{R}=-\frac{3}{10} \frac{T}{R}
$$

i.e., $\Delta T / R$ grows linearly with $T / R$. The correction to the recombination time $\Delta T / R$ reaches maximum for $T / R \approx 2$ when the duration of the cycle is such that the two clouds at their maximum separation stop overlapping. At longer cycle times $T / R>2$, both $D_{1}$ and $D_{2}$ become constants and $\Delta T / R$ starts decreasing inversely proportional to $T$ :

$$
\frac{\Delta T}{R}=-\frac{4}{5}\left(\frac{T}{R}\right)^{-1}
$$


This behavior has simple physical explanation. The difference between the corrections to the propagation velocities of the clouds $\Delta \kappa$ is due to the nonlinear interaction between the clouds and is accumulated only when the clouds overlap (see the definition of $D_{1}$ Eq. (23)). For short cycle times $T / R<2$, when the clouds overlap during all the cycle, the nonlinear effects are accumulated during all times and $\Delta \kappa \propto T^{2}$. The parabolic phase described by the coefficient $g(T)$ grows linearly with time $T$, so the correction to the recombination time $T$ is a growing function of the cycle time $T$. For $T>R$, when the clouds fully separate during the cycle, $\Delta \kappa$ is at its maximum possible value and stops growing further. The quadratic phase profile of each cloud, on the other hand, keeps growing as a function of time, i.e., $g$ becomes larger, thus resulting in the decrease of $\Delta T$.

The dependence of the shift in the recombination time $\Delta T / R_{0}$ on the cycle time $T / R_{0}$ is shown in Fig. 6 for $R_{0}=500$ and $p=5$. The maximum cycle times shown in Fig. 6 correspond to the maximum separation of the clouds equal to about ten their diameters. The dots are the results obtained by direct numerical solution of the Gross-Pitaevskii equation (6) and the solid line is given by Eq. (48). The optimized contrast of the interference fringes $V$ at the recombination time $T+\Delta T$ for the parameters of Fig. 7 is shown in Fig. 7 . The dots correspond to the numerical solution of the Gross-Pitaevskii equation and the solid line is given by Eq. (47). For comparison, the dashed line shows the contrast at the nominal recombination time $T$ given by Eqs. (43) and (40). The lowest values of the optimized contrast $V \approx 0.5$ correspond to intermediate cycle times $T / R_{0} \approx 2$ when the maximum separation between the two clouds is equal to their size. Both increasing and decreasing the cycle time $T$ improves the contrast.

Figs. 6 and (7) show that the operation of the atom Michelson interferometer with the optimization of the recombination time is possible both in the limit $T / 2 R_{0} \leq 1$ when the clouds overlap during all the cycle and in the opposite limit $T / 2 R_{0} \gg 1$ when the clouds are separated most of the time.

\section{B. Nonzero initial parabolic phase $g_{0} \neq 0$}

Performing an interferometric cycle with nonzero initial values of the parabolic phase $g_{0}$ considerably improves the coherence as compared to the case $g_{0}=0$ provided the sign of $g_{0}$ is the same as that of the nonlinearity $p$. The nonzero initial parabolic phase can be acquired 
by relaxing the confinement frequency $\omega$ of the initial trap and letting the condensate evolve for some time before the start of the interferometric cycle. Dynamics of the BEC in timedependent parabolic traps in Thomas-Fermi limit has been extensively analyzed (see, e.g. [14-16]). In the case of 1D expansion corresponding to our situation, the evolution of $g(\tau)$ and $r=R(\tau) / R(0)$ is described by the set of equations

$$
\begin{aligned}
& \frac{d^{2}}{d \tau^{2}} r=-\omega^{2}(\tau) r+\frac{\omega^{2}(0)}{r^{2}}, \\
& g=\frac{d}{d \tau} \ln r
\end{aligned}
$$

where $\omega(\tau)$ is the trap frequency. The exact value of $g$ depends on the detailed time dependence of $\omega(\tau)$. Changing $\omega(\tau)$ adiabatically slowly leaves the phase of the condensate flat, i.e. $g=0$. Since we are interested in the maximum possible value of $g$, we shall consider the limit when the trap frequency is relaxed very fast so that $\omega(\tau)=0$ for $\tau>0$. In this limit, $g$ is given by the relation (the condensate's initial phase in the trap is zero):

$$
g(r)=\left(\frac{3 p}{R_{t r}^{3}}\right)^{1 / 2}\left(\frac{r-1}{r^{3}}\right)^{1 / 2},
$$

where $R_{t r}$ is the initial radius of the condensate in the trap. An extra factor of two in Eq. (52) as compared to Eq. (32) is due to the fact that the initial condensate is normalized to one whereas the two propagating clouds are normalized to $1 / 2$.

For the given value of $R_{t p}, \mathrm{~g}$ is maximum for $r=3 / 2$. The final size of the condensate after the expansion is the initial size $R_{0}$ of the propagating clouds in the interferometric cycle, i.e., $R_{0} / R_{t r}=3 / 2$. The maximum possible value of $g_{0}$ is thus given by the relation

$$
g_{0, \max }=\left(\frac{3 p}{2 R_{0}^{3}}\right)^{1 / 2} .
$$

In the following we will use the value $g_{0}=s g_{0, \max }$ where the coefficient $0 \leq s \leq 1$ accounts for relaxing the trap with finite speed.

The correction to the recombination time $\Delta T / R$ given by the equation (46) with $\beta=0$ takes the form

$$
\frac{\Delta T}{R}=\frac{-2 D_{1}(T / R)}{(3 / 4)(T / R)+D_{2}(T / R)+s(3 R / 2 p)^{1 / 2}}
$$

If the parameter $(R / p)$ is large, which is typically the case, the corrections to the recombination time are small and the contrast is high. This is illustrated by Fig. 8 which shows the shift in the recombination time $\Delta T / R$ as a function of the cycle time $T / R$ using Eq. (54). 
The solid line corresponds to $s=0$ when the condensate does not have initial parabolic phase. The dashed curve gives $\Delta T / R$ for $s=0.2$, when the condensate has been allowed to acquire initial parabolic phase. The dots are the results of a numerical solution of the GPE with $s=0.2$. Figure 8 demonstrates that the shift in the recombination time is considerably smaller when the condensate is allowed to expand before the beginning of the cycle. Since the two harmonics have larger overlap at the optimal recombination time, the contrast in the interference fringes is larger when $g_{0} \neq 0$.

\section{Nonzero parabolic external potential $\beta \neq 0$}

Nonzero values of the parabolic external potential $\beta \neq 0$ can be due to environment or technical imperfections of an experimental apparatus. Equation (46) with $\beta \neq 0$ and $g_{0}=0$ yields

$$
\frac{\Delta T}{R}=-\frac{1}{4} \frac{8 D_{1}(\zeta)-\left(\beta R^{3} / p\right) \zeta^{2}}{3 \zeta / 4+D_{2}(\zeta)-\left(\beta R^{3} / p\right) \zeta}
$$

where $\zeta=T / R$. The influence of the parabolic potential on the operation of the atom Michelson interferometer is characterized by the parameter $b=\beta R^{3} / p$. Note that since the term with $\beta$ in the numerator of Eq. (55) is proportional to the square of the cycle time and the denominator grows linearly with time, even small values of $b$ for long enough cycles will always result in a complete loss of coherence.

In the limit of short cycle times $\zeta \ll 1$, Eq. (54) takes the form

$$
\frac{\Delta T}{R}=-\frac{\zeta}{4} \frac{3-b}{5 / 2-b} .
$$

Equation (56) is similar to Eq. (49) but the sign of $\Delta T$ can be both negative and positive depending on the value of $b$. The second difference is in that the coefficient multiplying $\zeta$ may become so large for positive values of $b \approx 5 / 2$, that coherence will be lost even for short cycle times. Negative values of $\beta$ are preferable because they ensure the operation of the interferometer at least for short times $T / R \leq 1$. If the value of $\beta$ is controlled at the level $b \ll 1$, the operation of the interferometer is possible for $\Delta T / R<1$ and any sign of $\beta$.

In the limit $\zeta>2$, Eq. (55) becomes

$$
\frac{\Delta T}{R}=-\frac{12 / 5-b \zeta^{2}}{(3-4 b) \zeta} .
$$


If $|b| \ll 1$, the optimized contrast will be high in the range

$$
2 \leq \frac{T}{R} \ll \frac{1}{|b|} .
$$

If $|b| \geq 1$, the coherence in general will be lost for $T / R>2$.

Additional limitations on the strength of the quadratic potential $\beta$ and the cycle time $T$ are due to the fact that the reflection pulses will not operate well if the relative change in the velocity of the atomic cloud $|\Delta v| / v_{0}$ exceeds about 0.1 . Using the fact that the nominal dimensionless velocity $v_{0}=1$ and the relation $|\Delta v|=(1 / 2)|\beta|(T / 2)^{2}$, one gets $|\beta| T^{2} \leq 0.8$.

\section{Recombination at a different wavelength}

The contrast of the interference fringes can be improved by conducting the recombination with optical pulses having different wavelength as compared to the splitting pulse to compensate for the change in the wave vectors of the moving clouds. The relative change in the wavelength of the recombining pulse $\Delta \lambda / \lambda$ as compared to the separation pulse is given by the expression (cf. Eq. (21))

$$
\frac{\Delta \lambda}{\lambda}=-\Delta \kappa=-\frac{\beta}{2} T^{2}+\frac{4 p}{R} D_{1}(T / R) .
$$

As has been discussed in the introduction, the repulsive nonlinearity results in the speeds $v$ of the moving harmonics $\psi_{ \pm}$being smaller than the speed $v_{0}$ imparted by the separation pulse. The recombination then should be performed with beams of larger wavelength. Similarly, for $\beta<0$ (a potential hump) $\Delta \lambda>0$ and for $\beta>0$ (a potential trough) $\Delta \lambda<0$. The optimized contrast is determined by the relation

$$
V \approx 1-\frac{3}{2}\left(\frac{\Delta x}{2 R}\right)^{2}\left[\ln \frac{2 R}{|\Delta x|}+2 \ln 2-\frac{1}{2}\right],
$$

where $\Delta x$ is the separation between the centers of the harmonics $\psi_{ \pm}$at the recombination time given by Eq. (22). In a typical situation, $|\Delta x| / R \ll 1$ and the optimized contrast is close to one.

\section{ACKNOWLEDGEMENTS}

This work was supported by the Defense Advanced Research Projects Agency (Grant No. W911NF-04-1-0043). 


\section{APPENDIX A: DYNAMICS OF THE BEC DUE TO THE OPTICAL PULSES}

The optical potential is used to split the initial zero-momentum BEC cloud at the beginning of the interferometric cycle into the two harmonics with the momenta \pm 1 , reverse their direction of propagation in the middle of the cycle and recombine them at the end. The optical pulses are short and sufficiently intense so that the dynamics of the condensate is dominated by the optical potential when the laser beams are on and the diffraction, relative displacements of the clouds and the nonlinearity can be neglected. A good quantitative description of the BEC dynamics can be obtained keeping only the lowest three harmonics with $n=0, \pm 1$ in Eq. (7). The set of Eq. (8) with these approximations reduces to

$$
i \frac{d}{d \tau}\left[\begin{array}{c}
\psi_{-1} \\
\psi_{0} \\
\psi_{1}
\end{array}\right]=\frac{1}{2}\left[\begin{array}{lll}
1 & \Omega & 0 \\
\Omega & 0 & \Omega \\
0 & \Omega & 1
\end{array}\right]\left[\begin{array}{l}
\psi_{-1} \\
\psi_{0} \\
\psi_{1}
\end{array}\right] .
$$

Solution of Eq. (A1) has the form

$$
\left[\begin{array}{l}
\psi_{-1}(\tau) \\
\psi_{0}(\tau) \\
\psi_{1}(\tau)
\end{array}\right]=\left[\begin{array}{lll}
A_{11} & A_{12} & A_{13} \\
A_{12} & A_{22} & A_{12} \\
A_{13} & A_{12} & A_{11}
\end{array}\right]\left[\begin{array}{l}
\psi_{-1}(0) \\
\psi_{0}(0) \\
\psi_{1}(0)
\end{array}\right]
$$

where

$$
\begin{aligned}
& A_{11}=\frac{1}{2}\left[\cos \frac{s \tau}{4}+e^{-i \tau / 4}-\frac{i}{s} \sin \frac{s \tau}{4}\right] \\
& A_{12}=-2 i \frac{\Omega}{s} \sin \frac{s \tau}{4} \\
& A_{13}=\frac{1}{2}\left[\cos \frac{s \tau}{4}-e^{-i \tau / 4}-\frac{i}{s} \sin \frac{s \tau}{4}\right], \\
& A_{22}=\cos \frac{s \tau}{4}+\frac{i}{s} \sin \frac{s \tau}{4}
\end{aligned}
$$

and $s=\sqrt{1+8 \Omega^{2}}$. Using Eq. (A2) it is straightforward to show that the momentum reversal of the moving BEC clouds $\psi_{ \pm 1} \rightarrow \psi_{\mp 1}$ can be achieved with a single pulse of duration $\tau_{p}=4 \pi$ and magnitude $\Omega_{p}=(3 / 8)^{1 / 2}$. The unitary evolution matrix corresponding to the momentum reversal pulse is of the form

$$
U_{ \pm 1 \leftrightarrow \mp 1}=\left[\begin{array}{ccc}
0 & 0 & 1 \\
0 & 1 & 0 \\
1 & 0 & 0
\end{array}\right] .
$$


Splitting of the zero-momentum cloud $\psi_{0}$ into the two harmonics $\psi_{ \pm 1}$ and the recombination (the inverse of the splitting) requires a double pulse sequence. The first pulse with $\Omega_{p}=$ $(1 / 8)^{1 / 2}$ and $\tau_{p}=2^{1 / 2} \pi$ is followed by a period of free evolution when the lasers are turned off for a time interval $\tau_{e v}=2 \pi$ and then followed by the second optical pulse with $\Omega_{p}=(1 / 8)^{1 / 2}$ and $\tau_{p}=2^{1 / 2} \pi$. The evolution matrix for the splitting sequence is given by

$$
U_{0 \leftrightarrow \pm 1}=\left[\begin{array}{ccc}
-\frac{1}{2} \exp (-i \pi / \sqrt{2}) & \frac{1}{\sqrt{2}} & \frac{1}{2} \exp (-i \pi / \sqrt{2}) \\
\frac{1}{\sqrt{2}} & 0 & \frac{1}{\sqrt{2}} \\
\frac{1}{2} \exp (-i \pi / \sqrt{2}) & \frac{1}{\sqrt{2}} & -\frac{1}{2} \exp (-i \pi / \sqrt{2})
\end{array}\right],
$$

(irrelevant common phase has been omitted).

[1] J. Reichel, Appl. Phys. B. 75, 469 (2002).

[2] M. Horikoshi and K. Nakagawa, Appl. Phys. B. 82, 363 (2005).

[3] W. Hansel, P. Hommelhoff, T. Hansch, and J. Reichel, Nature 413, 498 (2001).

[4] L. A. L. Feenstra and J. Schmiedmayer, General Relativity and Gravitation 36, 2317 (2004).

[5] S. Du, M. Squires, Y. Imai, L. Czaia, R. Saravanan, V. Bright, J. Reichel, T. Hansch, and D. Anderson, Phys. Rev. A. 70, 053606 (2004).

[6] Y. Shin, M. Saba, T. Pasquini, W. Ketterle, D. Pritchard, and A. Leanhardt, Phys. Rev. Lett. 92, $050405(2004)$.

[7] T. Schumm, S. Hofferberth, L. Andersson, S. Wildmermuth, S. Groth, I. Bar-Joseph, J. Schmiedmayer, and P. Kruger, Nature Physics 1 (2005).

[8] Y. Wang, D. Anderson, V. Bright, E. Cornell, Q. Diot, T. Kishimoto, M. Prentiss, R. Saravanan, S. Segal, and S. Wu, Phys. Rev. Letts. (2005).

[9] O. Garcia, B. Deissler, K. J. Hughes, J. M. Reeves, and C. A. Sackett, Physical Review A (Atomic, Molecular, and Optical Physics) 74, 031601 (2006).

[10] S. Wu, Y. Wang, Q. Diot, and M. Prentiss, Phys. Rev. A. 71, 043602 (2005).

[11] M. Olshanii and V. Dunjko, Interferometry in dense nonlinear media and interaction-induced loss of contrast in microfabricated atom interferometers (2005), URL arXiv.org:cond-mat/ 0505358.

[12] M. Horikoshi and K. Nakagawa, Phys. Rev. A. p. 031602 (2006).

[13] S. Wu, E.J.Su, and M. Prentiss, Eur. Phys. J. D 35, 111 (2005). 
[14] Y. Castin and R. Dum, Phys. Rev. Letts. 77 (1996).

[15] F. Dalfovo, C. Minniti, S. Stringari, and L. Pitaveskii, Phys. Letts. A. 227 (1997).

[16] Y. Kagan, E. Surkov, and G. Shlyapnikov, Phys. Rev. A. 55 (1997). 
FIGURES 


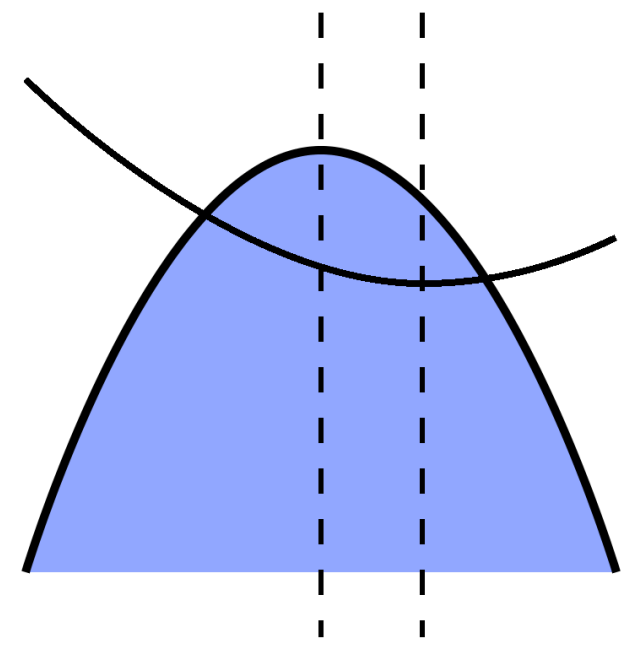

FIG. 1: The density and phase of the $\psi_{+}$harmonic before the recombination. 


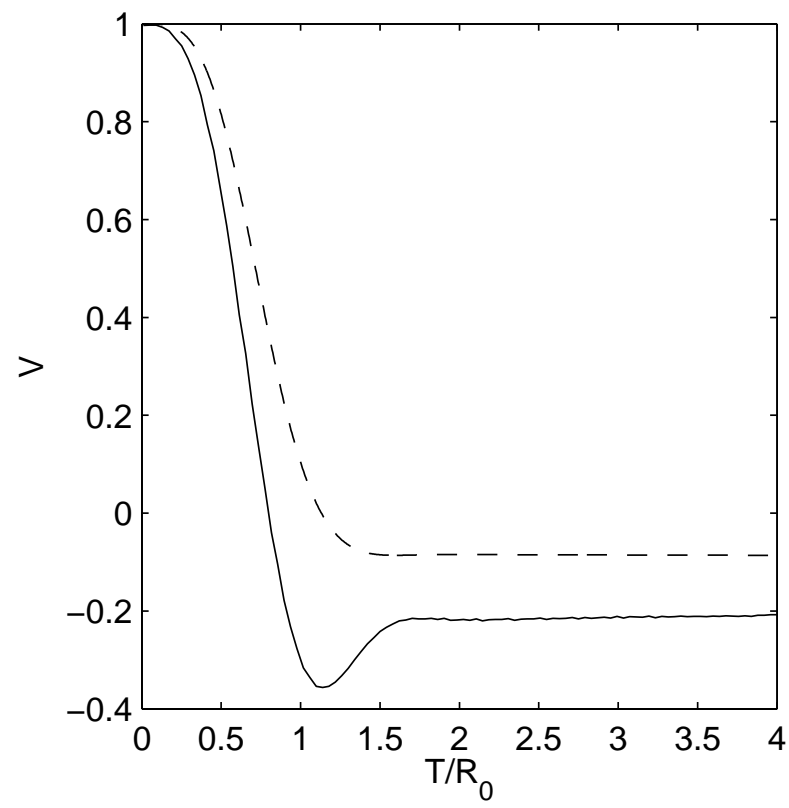

FIG. 2: The contrast $V$ versus the cycle time $T / R_{0}$ for $R_{0}=500, p=5, \alpha=0, g_{0}=0$ and $\beta=0$. 


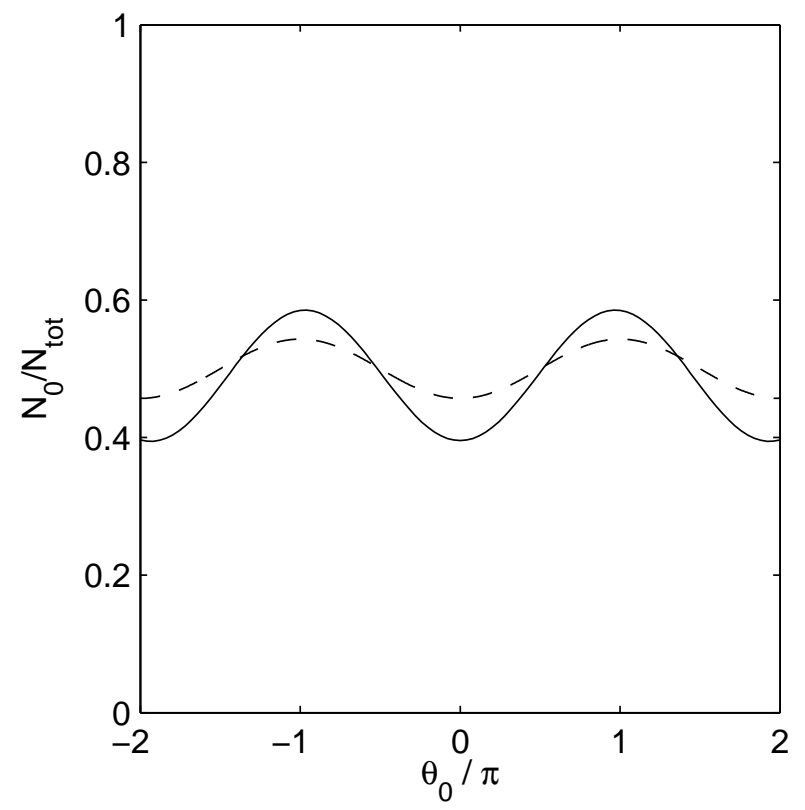

FIG. 3: Relative population of the zeroth-momentum harmonic $N_{0} / N_{\text {tot }}$ versus the relative accumulated phase $\theta_{0}=-\alpha T^{2} / 2$ for $T=2000$. Other parameters (except nonzero values of $\alpha$ ) are as in Fig.2. 


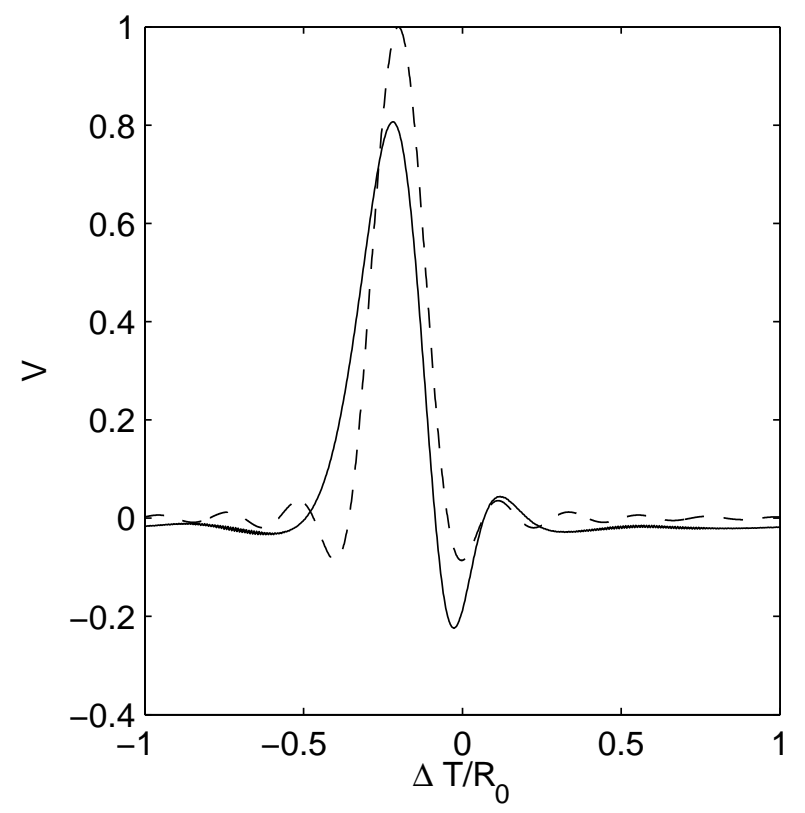

FIG. 4: The contrast $V=2 N_{0} / N_{\text {tot }}-1$ as a function of $\Delta T / R_{0}$. 


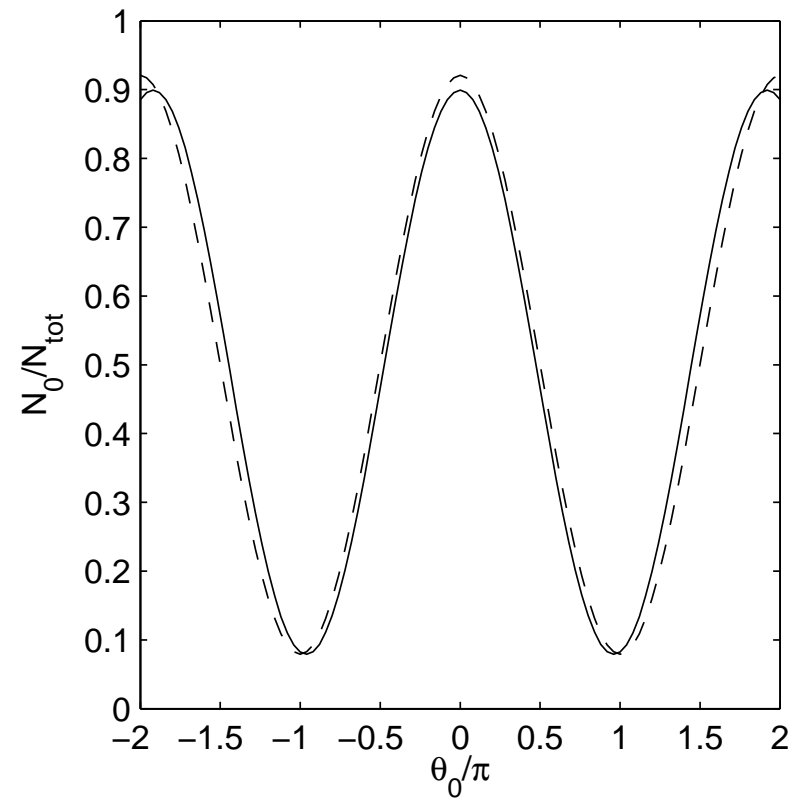

FIG. 5: Relative population of the zero-order harmonic $N_{0} / N_{\text {tot }}$ versus the relative accumulated phase shift $\theta_{0}$ for $T / R_{0}=-0.2$. All other parameters are the same as for Fig. 3 


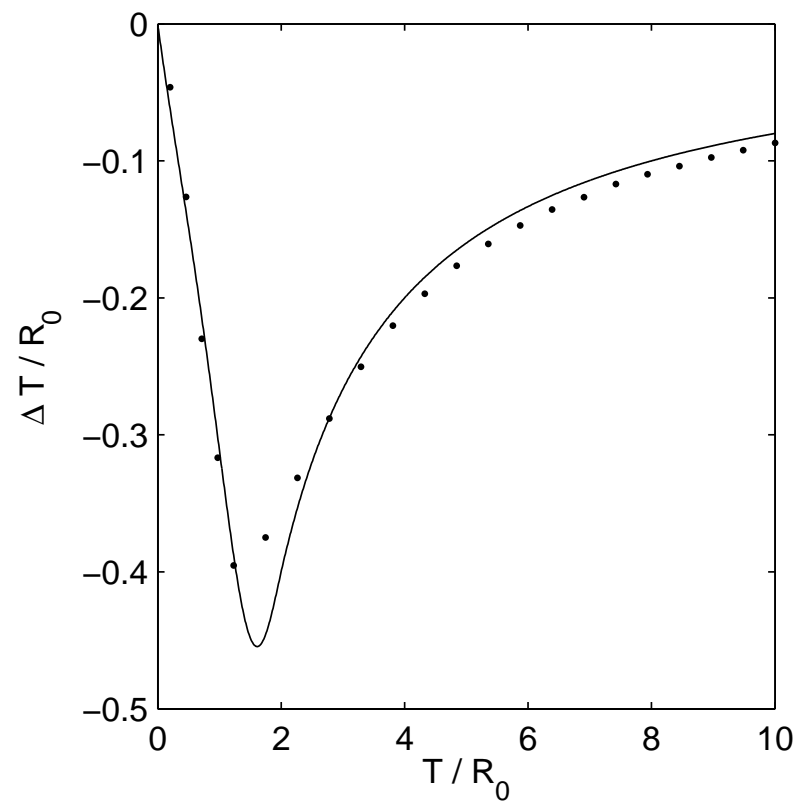

FIG. 6: The shift in the recombination time $\Delta T / R_{0}$ as a function of the nominal recombination time $T / R_{0}$ for $R_{0}=500$ and $p=5$. The dots are the results of the numerical solution of the GPE and the solid line is the analytical model. 


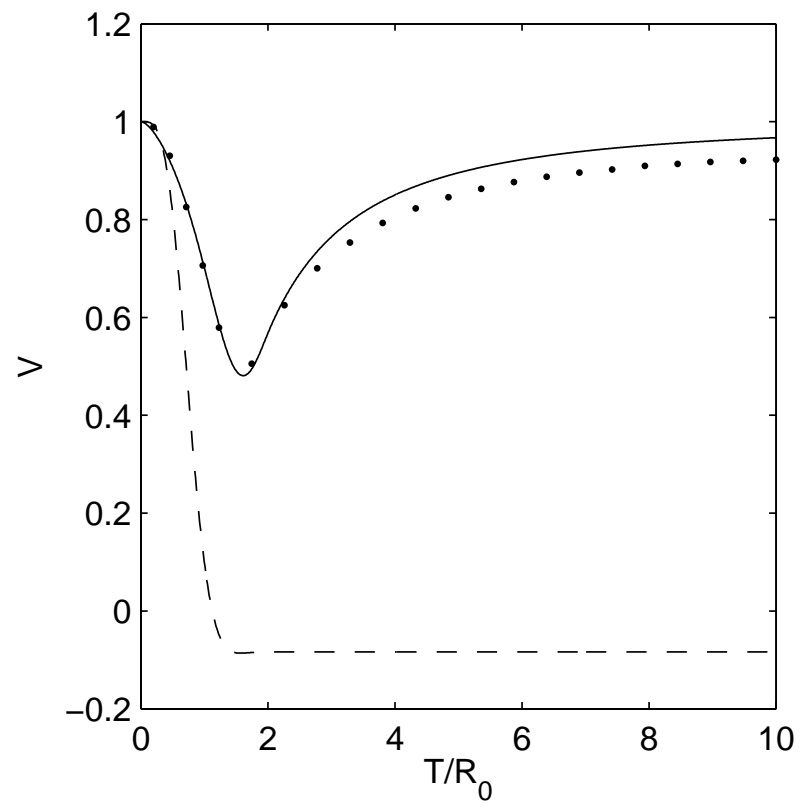

FIG. 7: Optimized contrast of the interference fringes $V$ for the parameters of Fig. 6 . The dots are the results of the numerical solution of the GPE and the solid line is the analytical model. The dashed line is the contrast at the nominal recombination time. 


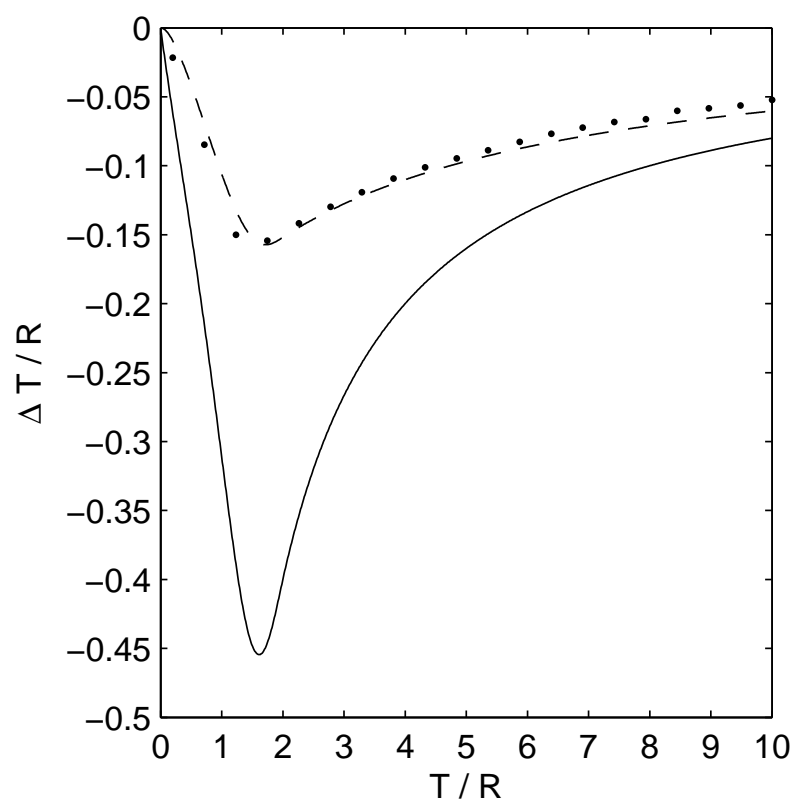

FIG. 8: The shift in the recombination time $\Delta T / R$ as a function of the cycle time $T / R$ given by Eq. (54) for $s=0$ (solid) and $s=0.2$ (dashed curve). The dots are the results of a numerical solution of the GPE. For all three curves $R / p=100$. 